\title{
Applications of State of the Art Infrared Chemical Imaging Technology
}

\author{
Norman A. Wright, John A. Seelenbinder, Ellen V. Miseo \\ Digilab, 68 Mazzeo Drive, Randolph, MA 02368 U.S.A.
}

Infrared Chemical Imaging is a fairly young technique, with the first commercial system available less than years ago. In that time period, tremendous interest has been shown for the applications of this technology, from its start with applications in the biological field to material and pharmaceutical analysis. Samples in general are not homogeneous with tissue and cellular samples especially good examples of non-uniformity with spatially separated areas of chemical difference. The great utility of infrared imaging is the ability to collect simultaneously both spatial and spectral information that results in both high fidelity images and high signal-to-noise spectra.

The use of focal plane array detectors to sense infrared radiation and generate thermal images is a technology that has developed over the past 15 years. Focal plane arrays detectors are basically of two types, uncooled and cooled. The uncooled technologies are currently receiving the bulk of the development funding and these materials have the potential to be inexpensive to manufacture and very rugged. Unfortunately, either the wavelength response is inadequate to determine chemical spectral signatures or the sensitivity is too low currently for a spectrochemical application. In addition they are inherently slow devices based on thermal response with the readout is limited to video rates. The only array detector currently manufactured that will meet the performance requirements of a spectroscopic measurement system, from both response and sensitivity criteria, is made from $\mathrm{HgCdTe}(\mathrm{MCT})$.

The essential technology, the infrared focal plane array, has not changed during the last 15 years because of the limited commercial demand and availability of these devices. The majority of the published studies before this year have used a "Javelin" 64 x 64 pixel MCT detector, which was not designed for spectroscopy but for one-time use in an antitank missile. This "Javelin detector" has a number of design parameters that limit its application in a laboratory spectroscopic environment. The readout on this detector is a rolling mode readout that limits the duty cycle to about 3\% and large amounts of potentially useable data are "thrown away."

With the experience gained in using the Javelin FPA, it was possible to design an FPA family better suited to FT-IR spectroscopy. This family of mid-infrared focal plane arrays (FPAs) is available in pixel formats from $16 \times 16$ up to $128 \times 128$. Focalplane arrays to formats of $64 \times 64$ pixels can be used in conjunction with a conventional rapid-scan Fourier transform spectrometer (FT-IR), and consequently provide very fast data acquisition times. These two advantages lead to collect data in very small time slices, and the ability to conduct experiments in a kinetic type collect mode[1]. These applications will be discussed. 
With the introduction of next generation detector technology specifically designed for spectroscopy, improved signal-to-noise performance has allowed a wider range of analyses. In addition, both microscopic and macroscopic applications have been developed to address a range of samples. The use of ATR measurements has significantly widened the applications of infrared imaging[2], examples will be shown.

The technology and integration of hardware components has evolved so that this spectroscopic imaging system can be viewed, instead of as a specialized research tool as that of an everyday problem-solving tool.

\section{References}

1) Koenig, Jack L.; Ribar, Travis; Shin, Eun-Mi; Wright, Norman, “Analysis of binary solvent diffusion into cross-linked poly (butadiene) using fast scan Fourier transform infrared imaging spectroscopy”, Appl Spectrosc, 55, p 1573-1579, (2001) FTS7000/Stingray/Lancer

2) Marcott, C.; Sommer, A.J.; Story, G.M.; Tisinger, L.G. "Attenuated total internal reflection infrared mapping microspectroscopy using an imaging microscope" Appl Spectrosc , 55, 252-256, 2001 6000/Stingray/MCT array 